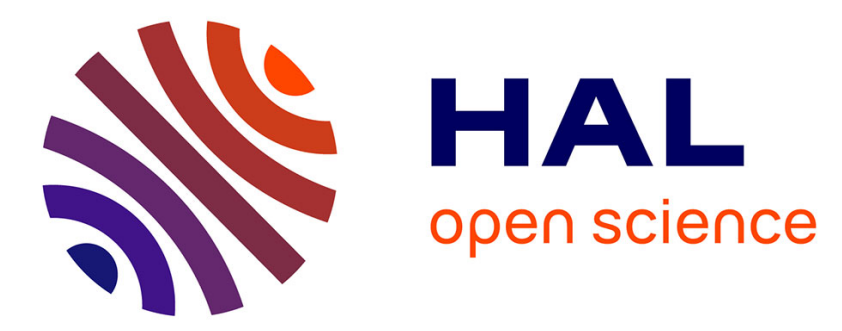

\title{
Heat conduction and thermal conductivity of 3D cracked media
}

\author{
M.N. Vu, S.T. Nguyen, M.H. Vu, Anh Minh A.M. Tang, V.T. To
}

\section{To cite this version:}

M.N. Vu, S.T. Nguyen, M.H. Vu, Anh Minh A.M. Tang, V.T. To. Heat conduction and thermal conductivity of 3D cracked media. International Journal of Heat and Mass Transfer, 2015, 89, pp.11191126. 10.1016/j.ijheatmasstransfer.2015.05.113 . hal-01271134

\section{HAL Id: hal-01271134 \\ https://hal-enpc.archives-ouvertes.fr/hal-01271134}

Submitted on 26 Apr 2018

HAL is a multi-disciplinary open access archive for the deposit and dissemination of scientific research documents, whether they are published or not. The documents may come from teaching and research institutions in France or abroad, or from public or private research centers.
L'archive ouverte pluridisciplinaire HAL, est destinée au dépôt et à la diffusion de documents scientifiques de niveau recherche, publiés ou non, émanant des établissements d'enseignement et de recherche français ou étrangers, des laboratoires publics ou privés. 


\title{
Heat conduction and thermal conductivity of 3D cracked media
}

\author{
M.N Vu ${ }^{1, *}$, S.T Nguyen ${ }^{1,}$, M.H Vu ${ }^{1,2}$, A.M Tang ${ }^{3}$, V.T To ${ }^{4}$ \\ ${ }^{1}$ R\&D Center, Duy Tan University, Da Nang, Viet Nam \\ ${ }^{2}$ CurisTec, 3 rue Claude Chappe, Parc d'affaire de Crécy, 69370 Saint-Didier-au-Mont-d'Or, France \\ ${ }^{3}$ Université Paris-Est, Laboratoire Navier (ENPC-IFSTTAR-CNRS) 77455 Marne la Vallée, France \\ ${ }^{4}$ Le Quy Don Technical University, 100 Hoang Quoc Viet, Hanoi, Vietnam \\ *Corresponding author: Email: vungocminh@dtu.edu.vn ; Tel.: +84 5113656109
}

\begin{abstract}
This study deals with the heat conduction within a medium containing cracks that are assumed to be perfect insulators. Multi-region boundary element approach is employed to obtain a boundary singular integral equation governing the steady state thermal transfer within this medium. This equation presents the temperature field within the whole cracked body as a function of temperature and rate of heat flow on the domain's boundary and temperature discontinuity across the cracks. For the particular case of an infinite domain under far-field condition, the temperature field solution is only a function of the cracks temperature's discontinuity. The basic problem of a single crack in an infinite domain is investigated and a closed-form solution is derived for a crack of elliptical plane from this analysis. This solution is the key issue to estimate the effective thermal conductivity of the whole domain by coupling with the classical homogenization schemes. The arbitrary crack form is covered up by using the excluded volume definition. Estimations of effective thermal conductivities stemming from diluted, differential and self-consistent approaches are compared to numerical solution obtained by the finite volume method that is available in literature. This comparison shows that the self-consistent scheme is the most appropriate model to estimate the thermal conductivity of materials containing cracks.
\end{abstract}

Keywords: thermal conductivity, crack, insulator, $M R$-BEM, self-consistent.

\section{Introduction}

The effects of cracks on the thermal conductivity have been investigated on various materials such as optical coatings [1], graphite materials [2], ceramics [3], thermal barrier coatings [4,5,6], fibre-reinforced composites [7], clay [8] and among others. These studies show that the thermal conductivity of materials decreases with the presence of cracks due to the very low thermal conductivity of air void created by cracks. The presence of cracks was evidenced by micrograph [3,5], topological sensitivity $[9,10]$ and was quantitatively characterized to interpret the results on thermal conductivity [6]. 
X-ray micro-tomography can be considered as a powerful tool to monitor the 3D spatial distribution of cracks in materials [11].

To model the heat conduction within materials containing cracks of complex geometry and to take into account the interaction between the cracks, the numerical method imposes naturally. The use of volume discretisation methods, such as finite element method [12,13], finite volume method [14,15,16,17] is confronted to a major difficulty in mesh generation process when the domain contains a numerous cracks of randomly geometries. Contrariwise, boundary element method (BEM) presents an important advantage in mesh reduction $[18,19,20]$. In practice, the classical BEM becomes also inefficiently for high crack density by the fact of complicated numerical implementation compared to standard BEM for the intact domain.

Atalay et al [21] employed the multi-region boundary element method (MR-BEM) to modeling the steady-state heat conduction in a heterogeneous system constituted by several homogeneous piecewise functions. This technique has been also applied for other types of heterogeneous media such as materials in the presence of cracks, in particular in the linear fracture mechanics [20,22]. However, only a single or a few cracks are treated and the crack intersection is not considered in this application field. Recently, Pouya [24] and Pouya and $\mathrm{Vu}[23,25]$ proposed the closed-form expressions of mass exchange between matrix and cracks at crack intersections for fluid flow modeling. Then, Vu [26] associated this advanced theoretical formulation with MR-BEM to develop the integral boundary equation and its numerical resolution for modeling the fluid flow within a porous domain containing numerous intersecting cracks. This method is now employed to study heat conduction within the cracked media where the crack filled by air whose conductivity is neglected in compared to one of its surrounded material. Therefore, the cracks could be considered as a barrier to the heat flow that is naturally contrary to the case of fluid flow where the crack is more conductive than the porous embedding matrix.

The present work focuses on the steady-state heat flow within a three dimension finite domain containing numerous cracks and its effective thermal conductivity. We make assumption that crack has zero thickness and an infinitely cross thermal resistance, i.e. it acts as a barrier to heat flow. Therefore, the rate of heat flow vanishes on the crack surfaces in its normal direction; and there exists a temperature discontinuity across it. MR-BEM is employed to derive the boundary integral equation that governs heat conduction through the whole domain presenting a high crack density. For this end, the domain is split into several sub-domains by existing cracks and fictitious surfaces, such that a fictitious surface does not intersect cracks and other fictitious surfaces. The unknown temperature on the each face of crack is considered as the boundary condition for the sub-domains. Boundary integral formulation for fluid flow is written for each 
sub-region and then assembled to obtain only one potential solution for the whole body. In this solution, the temperature field is expressed as function of temperature and heat flow on the domain's boundary and temperature discontinuity across the cracks.

This theoretical solution is used subsequently to estimate of effective thermal conductivity of the material with the presence of void cracks. For this purpose, infinite domain is considered. The closed-form solution of temperature discontinuity is derived from the singular integral equation writing for the case of a crack with elliptical shape. The arbitrary crack shape is covered in this study by having recourse to excluded volume definition $[27,28,29,30]$. The irregular shape of cracks could be also taken into account by analysing the strength of the heat flux singularity in vicinity of crack front [31]. The analytical solution of temperature discontinuity allows estimating the effective thermal conductivity in the framework of various homogenization schemes: dilute, differential and self-consistent. The details of these techniques for thermal properties of porous geomaterial were synthesized in Do et al. [32,33,34], To et al. [35,36], Nguyen [37] and Chen [38]. Effective properties of micro-cracked viscoelastic materials are also successfully estimated by coupling the solution of a single crack in infinite domain and the homogenization schemes (Nguyen et al. [39,40]). The numerical solution obtained by Bogdanov et al. [14], based on the finite volume approach, is finally used to evaluating the accuracy of these three schemes for the material that contains insulating cracks.

\section{Notations}

- $\Omega: \quad$ micro-cracked domain

- $\quad \Gamma, \Gamma_{i}$ : cracks set and crack number $i$

- $\lambda$ : thermal conductivity tensor of matrix without cracks

- $\lambda^{e f f}$ : effective thermal conductivity tensor of cracked media

- $\lambda$ : thermal conductivity of isotropic matrix

- $\lambda^{\text {eff: }}$ effective thermal conductivity of isotropic cracked media

- $q(\underline{x})$ local rate of heat flow at a point $\underline{x}$

- $\quad T(\underline{x})$ : local temperature at a point $\underline{x}$

- $\underline{s}\left(s_{1}, s_{2}\right)$ : curvilinear abscise of smooth crack surface

- $\quad[[T(\underline{z})]]=T^{+}(\underline{z})-T^{-}(\underline{z})$ : temperature discontinuity across crack surface

- $c, c_{i}$ : boundary factors of whole and sub-domain respectively

- $G$ : fundamental solution

- $\quad \rho$ : $\quad$ crack density 
- $\quad v$ : dimensionless crack density

- $Q$ : $\quad$ average rate of heat flow

- $\underline{G}$ : average temperature gradient

- $\underline{A}$ : far-field temperature gradient

- $\nabla: \quad$ gradient operator

- $\Delta: \quad$ Laplace operator

- $\quad \nabla .:$ divergence operator

- $\delta: \quad$ Dirac distribution

- $\partial_{s} f: \quad$ derivation of function $f$ with respect to variable $s$

- $\quad \underline{a} . \underline{b}: \quad$ the scalar product of two vectors $\underline{a}$ and $\underline{b}$

- $|\boldsymbol{A}|: \quad$ the determinant of matrix $\boldsymbol{A}$

- $\quad \boldsymbol{A} \underline{a}: \quad$ the product operator between matrix $\boldsymbol{A}$ and vector $\underline{a}$

\section{Thermal conduction equations}

Let us consider a three-dimensional domain $\Omega$ embedding $m$ cracks numbered by $j$ and denoted by $\Gamma_{j}(j=1, m)$ (Fig. 1). In the mathematical model, cracks are represented by a smooth surface function $\underline{z}_{j}(\underline{s})$ from $\mathbb{R}^{3} \rightarrow \mathbb{R}^{2}$, where $\underline{s}=\left(s_{1}, s_{2}\right)$ is the curvilinear parameters. $\Gamma$ designates the set of cracks $\Gamma=\cup \Gamma_{j}$. The matrix without cracks corresponds to $\Omega-\Gamma$ that is assumed to be homogeneous with a conductivity tensor $\lambda$.

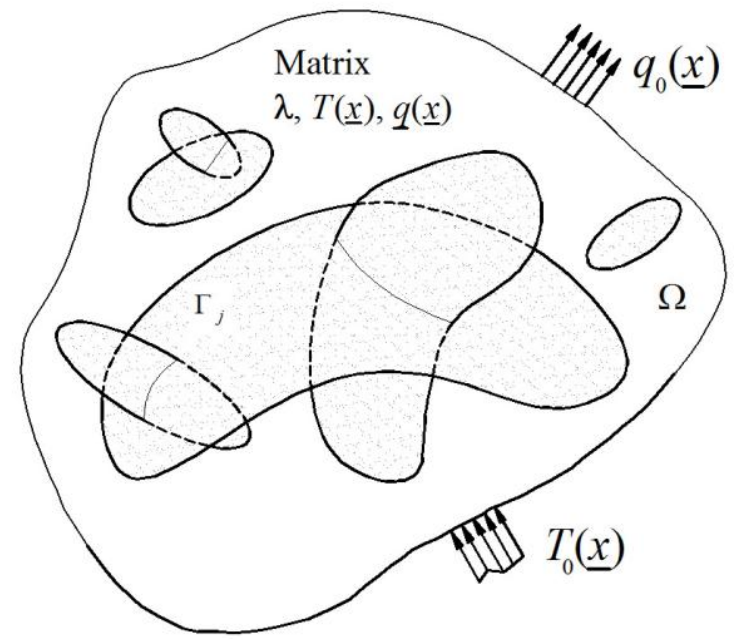

Fig. 1: Heat conduction within a medium embedding cracks.

The heat conduction in the matrix is governed by Fourier's law: 
$\forall \underline{x} \in \Omega-\Gamma \quad \underline{q}(\underline{x})=-\lambda . \nabla T(\underline{x})$

where $q(\underline{x})$ and $T(\underline{x})$ are local rate of heat flow and temperature fields, respectively.

The conservation of energy in the matrix reads:

$\forall \underline{x} \in \Omega-\Gamma \quad \nabla \cdot \underline{q}(\underline{x})=0$

Substituting (1) into (2) yields Laplace's equation for the case of an isotropic matrix, i.e $\lambda=\lambda \delta$ :

$$
\forall \underline{x} \in \Omega-\Gamma \quad \Delta T(\underline{x})=0
$$

Solving this equation gives the solution of steady state heat diffusion in the matrix $\Omega-\Gamma$. This solution must verify the condition on the cracks and the boundary conditions. As the cracks are non-conducting and act as barriers to thermal flow, the conditions on the crack surfaces are given as:

$\forall \underline{x} \in \Gamma \quad q(\underline{x}) \cdot \underline{n}(\underline{x})=0$ or $\nabla T(\underline{x}) \cdot \underline{n}(x)=0$

where $\underline{n}(\underline{x})$ is the unit normal vector at point $\underline{x}$ on cracks.

Two boundary conditions, namely Dirichlet and Neumann conditions are prescribed respectively on $\partial \Omega_{1}, \partial \Omega_{2}$ of the domain boundary $\partial \Omega\left(\partial \Omega=\partial \Omega_{1}+\partial \Omega_{2}\right)$ :

$\forall \underline{x} \in \partial \Omega_{1} \quad T(\underline{x})=T_{0}(\underline{x})$

$\forall \underline{x} \in \partial \Omega_{2} \quad-\lambda \nabla T(\underline{x}) \cdot \underline{n}(\underline{x})=q_{0}(\underline{x})$

\section{Potential solution}

\subsection{Multi-region boundary elements approach}

This sub-section is devoted to establish a singular integral equation for the temperature field in the whole domain $\Omega$ that satisfies the Laplace equation (3), the condition on crack surfaces (4) and the boundary conditions (5), (6). For this purpose, the MR-BEM is had recourse. As mentioned in the introduction section, this approach exhibits a great advantage in mesh generation term for three-dimensional domain in the presence of numerous surfaces of discontinuities.

The domain $\Omega$ is first partitioned into $n$ sub-domains $\Omega_{i}$ by the fictitious boundaries that pass through the crack parts, as seen in Fig. 2. A crack $\Gamma$ of normal $\underline{n}$ is constituted by 
two parallel surfaces $\Gamma^{+}$and $\Gamma^{-}$with two normal vectors $\underline{n}^{+}, \underline{n}^{-} \quad\left(\underline{n}^{+}=-\underline{n}^{-}=\underline{n}\right)$ (Fig. 3 ). On the crack surface, the temperature is discontinuous between its two faces and the normal heat rate vanishes, i.e. $q \cdot \underline{n}=0 .[[T(z)]]=T^{+}(z)-T(z)$ designates the temperature discontinuity across the crack surface. A fictitious surface $\Gamma^{\prime}$ is assumed to have two coincidental faces $\Gamma^{\prime+}$ and $\Gamma^{\prime-}$ through which the temperature and the heat rate are continuous. As showed latterly, the fictitious surfaces appear only in the developed step equations but have no contribution in the final boundary integral equation for the whole domain.

The boundary $\partial \Omega^{i}$ of subdomain $\Omega_{i}$ could be consisted of a portion of the boundary of whole domain denoted by $\partial \Omega_{i}^{s}, n_{1 i}$ positive crack surfaces, $n_{2 i}$ negative crack surfaces, $n_{3 i}$ positive fictitious surfaces and $n_{4 i}$ negative fictitious surfaces. The subdomains, nonconnected to the boundary $\partial \Omega$, are bounded only by the cracks and or fictitious surfaces.

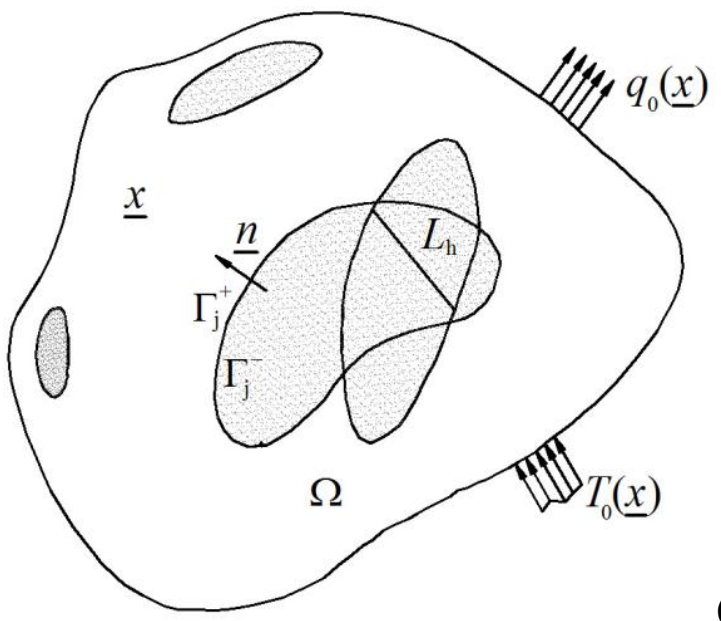

(a)

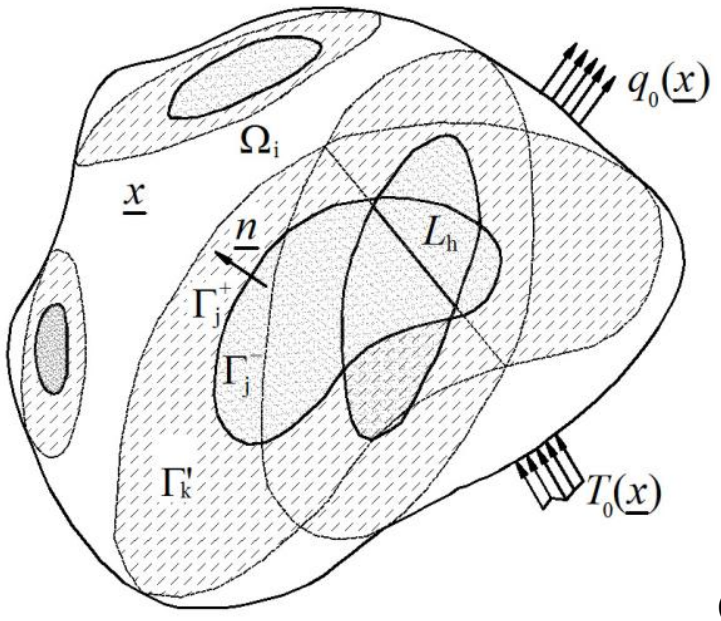

Fig. 2: MR-BEM approach to study heat conduction within cracked media

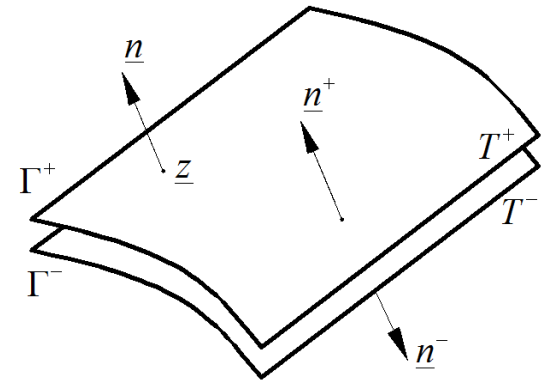

Fig. 3: Temperature discontinuity on the crack surface

As sub-domain $\Omega_{i}$ does not contain the crack, therefore, equation (3) governs the heat conduction within it. Applying the BEM procedure [18,19,20] for this Laplace's equation yields: 
$\forall \underline{\xi} \in \Omega_{\mathrm{i}} \quad c_{i}(\underline{\xi}) T(\underline{\xi})=\int_{\partial \Omega_{i}} T(\underline{x}) \nabla G(\underline{x}, \underline{\xi}) \cdot \underline{n} d s-\int_{\partial \Omega_{i}} G(\underline{x}, \underline{\xi}) \nabla T(\underline{x}) \cdot \underline{n} d s$

where $\underline{n}$ is the normal to the subdomain boundary $\partial \Omega_{i}$;

$c_{i}(\xi)$ a coefficient that depends on the position of $\xi$ relative to the subdomain $\Omega_{i}: c_{i}(\xi)=1$ if $\underline{\xi} \subset \Omega_{i}-\partial \Omega_{i} ; c_{i}(\xi)=0$ if $\underline{\xi} \not \subset \Omega_{i}$ and $0<c_{i}(\xi)<1$ if $\underline{\xi} \subset \partial \Omega_{i}$. For the latter case when the field point on the boundary of subdomain, the coefficient $c_{i}$ depends on the local boundary geometry at this point and it takes the value of $1 / 2$ for smooth boundary. In general, this coefficient can be determined by the following equation:

$\forall \underline{\xi} \in \partial \Omega_{\mathrm{i}} \quad c_{i}(\underline{\xi})=\lim _{\varepsilon \rightarrow 0} \int_{S_{\varepsilon}} G(\underline{x}, \underline{\xi}) d x$

where $S_{\varepsilon}$ is an infinitesimal spherical surface of centre $\xi$ and radius $\varepsilon$ enclosed in the solid $\Omega_{\mathrm{i}}$.

The fundamental function $G(\underline{x}, \underline{\xi})$ and its derivations for three-dimensional steady-state heat transfer are expressed such as $[18,19,20]$ :

$$
\begin{aligned}
& G(\underline{x}, \underline{\xi})=\frac{1}{4 \pi r} \\
& \frac{\partial G(\underline{x}, \underline{\xi})}{\partial \underline{n}_{x}}=\frac{1}{4 \pi} \frac{\underline{n} x \cdot \underline{R}}{r^{3}} \\
& \frac{\partial^{2} G(\underline{x}, \underline{\xi})}{\partial \underline{n}_{x} \partial \underline{n}_{\xi}}=\frac{1}{4 \pi}\left[\frac{\underline{n}_{\xi} \cdot \underline{n}_{x}}{r^{3}}-3 \frac{\left(\underline{n}_{\xi} \cdot \underline{R}\right)\left(\underline{n}_{x} \cdot \underline{R}\right)}{r^{5}}\right]
\end{aligned}
$$

with $\underline{R}=\underline{\xi}-\underline{x} ; r=\|\underline{R}\|=\|\underline{\xi}-\underline{x}\|, \underline{n}_{x}$ and $\underline{n} \xi$ the normal to crack surface at $\underline{x}$ and $\xi$.

The integrals on the right hand side of equation (7) are the sum of this integral on the different parts of boundary of this subdomain, i.e. on the $\partial \Omega_{i}^{s}, n_{1 \mathrm{i}}$ positive fracture surface segments $\Gamma_{k}^{+}, n_{2 \mathrm{i}}$ negative fracture surface segments $\Gamma_{k}^{-}, n_{3 \mathrm{i}}$ fictitious positive lines $\Gamma_{k}^{++}$and $n_{4 \mathrm{i}}$ fictitious negative lines $\Gamma_{k}^{\prime-}$.

$$
\begin{aligned}
c_{i}(\underline{\xi}) T(\underline{\xi})=\int_{\partial \Omega_{i}^{s}} T(\underline{x}) \nabla G(\underline{x}, \underline{\xi}) \cdot \underline{n} d s-\int_{\partial \Omega_{i}^{s}} G(\underline{x}, \underline{\xi}) \nabla T(\underline{x}) \cdot \underline{n} d s \\
\quad+\sum_{n_{1 i}} \int_{\Gamma_{k}^{+}} T^{+}(\underline{x}) \nabla G(\underline{x}, \underline{\xi}) \cdot \underline{n}^{+} d s-\sum_{n_{1 i}} \int_{\Gamma_{k}^{+}} G(\underline{x}, \underline{\xi}) \nabla T^{+}(\underline{x}) \cdot \underline{n}^{+} d s
\end{aligned}
$$




$$
\begin{aligned}
& +\sum_{n_{2 i}} \int_{\Gamma_{k}^{-}} T^{-}(\underline{x}) \nabla G(\underline{x}, \underline{\xi}) \cdot \underline{n}^{-} d s-\sum_{n_{2 i}} \int_{\Gamma_{k}^{-}} G(\underline{x}, \underline{\xi}) \nabla T^{-}(\underline{x}) \cdot \underline{n}^{-} d s \\
& +\sum_{n_{3 i}} \int_{\Gamma_{k}^{+}} T(\underline{x}) \nabla G(\underline{x}, \underline{\xi}) \cdot \underline{n}^{+} d s-\sum_{n_{3 i}} \int_{\Gamma_{k}^{+}} G(\underline{x}, \underline{\xi}) \nabla T(\underline{x}) \cdot \underline{n}^{+} d s \\
& +\sum_{n_{4 i}} \int_{\Gamma_{k}^{-}} T(\underline{x}) \nabla G(\underline{x}, \underline{\xi}) \cdot \underline{n}^{-} d s-\sum_{n_{4 i}} \int_{\Gamma_{k}^{-}} G(\underline{x}, \underline{\xi}) \nabla T(\underline{x}) \cdot \underline{n}^{-} d s
\end{aligned}
$$

As a matter of fact, the field point $\xi$ can be located inside, outside or on the boundary of domain $\Omega_{i}$. The relative position of this point relative to the subdomain is represented by the factor $\mathrm{c}_{i}$. Therefore, we can repeat the equation (10) for all subdomains but for a field point $\xi$ to obtain $n$ boundary integral equations. The sum of these $n$ equations results in:

$$
\begin{aligned}
\sum_{i=1}^{n} c_{i}(\underline{\xi}) T(\underline{\xi}) & =\int_{\partial \Omega} T(\underline{x}) \nabla G(\underline{x}, \underline{\xi}) \cdot \underline{n} d s-\int_{\partial \Omega} G(\underline{x}, \underline{\xi}) \nabla T(\underline{x}) \cdot \underline{n} d s \\
& +\sum_{k=1}^{m} \int_{\Gamma_{k}}\left[T^{+}(\underline{x})-T^{-}(\underline{x})\right] \nabla G(\underline{x}, \underline{\xi}) \cdot \underline{n} d s
\end{aligned}
$$

To show how we obtain the equation (11), we consider a fictitious surface $\Gamma^{\prime}{ }_{\mathrm{ij}}$ that are shared by two subdomains $\Omega_{\mathrm{i}}$ and $\Omega_{\mathrm{j}}$. Across the fictitious surface, the temperature and its gradient or thermal flux rate are continuous, therefore, the seventh, eighth, ninth, tenth terms in the boundary integral equation writing at the field point $\xi$ for the subdomain $\Omega_{\mathrm{i}}$ will be eliminated by the same terms in the one writing for its adjacent subdomain $\Omega_{\mathrm{j}}$. Moreover, the rate of heat flow is none on the crack surface, thus the fourth and sixth terms in the boundary integral equation for each subdomain are vanished.

By noting $c=\sum_{i=1}^{n} c_{i}$, it is easy to see that the coefficient $c$ is always equal to 1 when the field point is inside the whole cracked domain. Therefore, the factor $c$ of the cracked domain $\Omega$ can be represented by one of the un-cracked domain bounded by $\partial \Omega$.

Introducing the temperature discontinuity across the cracks into equation (11) yields the boundary integral equation for temperature field within the cracked media:

$$
c(\underline{\xi}) T(\underline{\xi})=\int_{\partial \Omega} T(\underline{x}) \nabla G(\underline{x}, \underline{\xi}) \cdot \underline{n} d s-\int_{\partial \Omega} G(\underline{x}, \underline{\xi}) \nabla T(\underline{x}) \cdot \underline{n} d s
$$




$$
+\sum_{k=1}^{m} \int_{\Gamma_{k}}[[T(\underline{x})]] \nabla G(\underline{x}, \underline{\xi}) \cdot \underline{n} d s
$$

Let us consider now the particular case of an infinite medium surrounding cracks under the far-field condition $T_{\infty}(\underline{x})$. As the field point $\underline{\xi}$ always inside the domain, thus the boundary factor $c$ is equal to 1 . The equation (12) reduces to:

$$
T(\underline{\xi})=T_{\infty}(\underline{\xi})+\sum_{k=1}^{m} \int_{\Gamma_{k}}[[T(\underline{x})]] \nabla G(\underline{x}, \underline{\xi}) \cdot \underline{n}_{x} d s
$$

In heat conduction modelling point of view, numerical resolving equation (12) covers equation (13) by making a large domain's boundary, where the far-field condition $T_{\infty}(\underline{x})$ is prescribed, compared to cracked zone. The heat conduction solution is used then to calculate the effective thermal conductivity of cracked domain. However, the direct derivation of equation (13) that reduce the complication of numerical implementation in comparison with the one of equation(12), allows also the determination of thermal conductivity for a cracked zone [23].

The heat conduction solution (12) obtained under a singular integral equation form is suitable for both numerical simulation and analytical development. This provides an interesting material that can be useful for those who performs numerical simulation based on boundary element method by beneficing the mesh reduction from $3 \mathrm{D}$ to $2 \mathrm{D}$. Besides, this solution allows deriving the closed-form solution for heat conduction in some particular cases. As showed subsequently, the analytical resolution of equation (13) in the case of a single fracture within an infinite medium, coupling with homogenization methods affords us another ways to estimate the thermal conductivity of material with the presence of cracks.

\subsection{Closed-form solution of temperature discontinuity}

This section is devoted to derive the closed-form solution of temperature discontinuity across the a single crack with in an infinite matrix under far-field condition $\mathrm{T}_{\infty}(\xi)=\underline{A} . \xi$. Deriving equation (13) with respect to $\xi$ variable reads:

$$
\frac{\partial T(\underline{\xi})}{\partial \underline{n}_{\xi}}=\frac{\partial T_{\infty}(\underline{\xi})}{\partial \underline{n}_{\xi}}+\sum_{k=1}^{m} \int_{\Gamma_{k}}[[T(\underline{x})]] \frac{\partial^{2} G(\underline{x}, \underline{\xi})}{\partial \underline{n}_{\xi} \partial \underline{n}_{x}} d s
$$

Considering the field point $\xi$ located on the fracture surfaces at curvilinear parameter $\underline{s}$, the left hand side of equation (14) vanishes according to the condition (4). Thus, the equation (14) becomes: 


$$
\varphi(\underline{x})=\frac{1}{4 \pi} \sum_{j} \int_{\Gamma^{j}} \omega(\underline{s})\left[-\frac{\underline{n}(\underline{s}) \cdot \underline{n}(\underline{x})}{r^{3}(\underline{s}, \underline{x})}+3 \frac{(\underline{n}(\underline{s}) \cdot \underline{R}(\underline{s}, \underline{x}))(\underline{n}(\underline{x}) \cdot \underline{R}(\underline{s}, \underline{x}))}{r^{5}(\underline{s}, \underline{x})}\right] d s
$$

with

$$
\varphi(\underline{x})=\frac{\partial T_{\infty}(\underline{x})}{\partial \underline{n}_{x}} ; \omega(\underline{x})=[[T(\underline{x})]]
$$

This equation is a hyper--singular integral equation in which the temperature discontinuity on the fractures $\omega(\underline{x})$ is unknown function and the hypersingular kernel behaves as $r^{-3}$ at the singular point $(\underline{s}=\underline{x})$. The hyper-singular kernels in equation (15) are also arisen in symmetry Galerkin boundary element approximation for solving two or three-dimensional Laplace's equations. The algorithms based on limit process are presented in Sutradhar et al. [41] for evaluating these hyper-singular integrals. In such process, the integral involving derivatives of the fundamental solution are defined as limits from the interior.

In order to upscale the effective permeability, a basic problem is considered. This is to derive the temperature field around a single insulating crack within an infinite matrix under far-field condition. The crack is modelled by an elliptical form $D$ with halfdiameters designated by $d_{1}, d_{2}\left(d_{1} \geq d_{2}\right)$ and two corresponding principal directions $\underline{e}_{1}, \underline{e}_{2}$ denote elliptical fracture principal directions and its unit normal $\underline{e}_{3}$. A local coordinate system is defined such as the origin located at the centre of the crack and its axes parallel to $\underline{e}_{1}, \underline{e}_{2}$ and $\underline{e}_{3}$. The equation of ellipse $D$ in this coordinate system reads (Fig. 4):

$$
\underline{x} \cdot \boldsymbol{B} \cdot \underline{x}=1
$$

with $\underline{x} . \underline{e}_{3}=0$ or $\underline{x}=\left(x_{1}, x_{2}, 0\right)$ and

$$
\lambda_{1}=1 / d_{1}^{2}, \quad \lambda_{2}=1 / d_{2}^{2}, \quad \boldsymbol{B}=\lambda_{1} \underline{e}_{1} \otimes \underline{e}_{1}+\lambda_{2} \underline{e}_{2} \otimes \underline{e}_{2}
$$




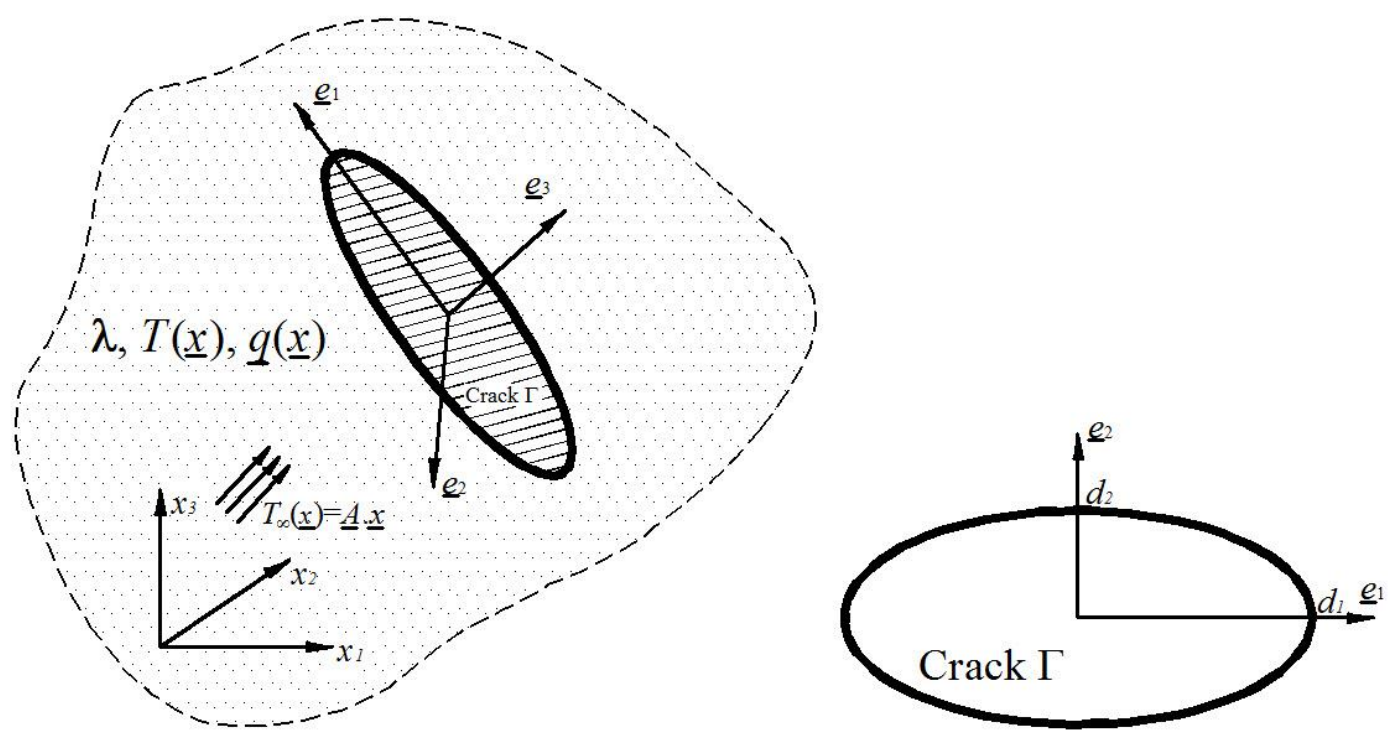

Fig. 4 A elliptical penny-shaped crack within an infinite matrix

For a single planar crack, $\underline{n}(\underline{s}) \cdot \underline{R}(\underline{s}, \underline{x})=\underline{n}(\underline{x}) \cdot \underline{R}(\underline{s}, \underline{x})=0$ and $\underline{n}(\underline{s}) \cdot \underline{n}(\underline{x})=1$, thus, equation (15) is rewritten as follows:

$$
\forall \underline{x} \in D \quad \varphi\left(x_{1}, x_{2}\right)=-\frac{1}{4 \pi} \int_{D} \frac{\omega\left(s_{1}, s_{2}\right)}{\left[\left(s_{1}-x_{1}\right)^{2}+\left(s_{2}-x_{2}\right)^{2}\right]^{3 / 2}} d s_{1} d s_{2}
$$

This equation is equivalent to:

$$
\forall \underline{x} \in D \quad-4 \pi \varphi\left(x_{1}, x_{2}\right)=\int_{D} \omega\left(s_{1}, s_{2}\right) \Delta_{s} \frac{1}{\left[\left(s_{1}-x_{1}\right)^{2}+\left(s_{2}-x_{2}\right)^{2}\right]^{1 / 2}} d s_{1} d s_{2}
$$

Since the temperature is continuous at the crack border, i.e. $\omega\left(x_{1}, x_{2}\right)$ subject to the following condition:

$\forall \underline{x} \in \partial D \quad \omega\left(x_{1}, x_{2}\right)=0$

Integrating by part and the condition (21) lead to:

$$
\forall \underline{x} \in D \quad 4 \pi \varphi\left(x_{1}, x_{2}\right)=\int_{D} \nabla \omega\left(s_{1}, s_{2}\right) \cdot \nabla \frac{1}{\left[\left(s_{1}-x_{1}\right)^{2}+\left(s_{2}-x_{2}\right)^{2}\right]^{1 / 2}} d s_{1} d s_{2}
$$

In the case that the far-field condition is a constant pressure gradient $\underline{A}$, i.e $T_{\infty}(\underline{x})=\underline{A} . \underline{x}$, hence, $\varphi(\underline{x})=\underline{A} \cdot \underline{e}_{3}$, equation (22) is rewritten as: 


$$
\forall \underline{x} \in D \quad 4 \pi \underline{A} \cdot \underline{e}_{3}=\int_{D} \nabla \omega_{3}\left(s_{1}, s_{2}\right) \cdot \nabla \frac{1}{\left[\left(s_{1}-x_{1}\right)^{2}+\left(s_{2}-x_{2}\right)^{2}\right]^{1 / 2}} d s_{1} d s_{2}
$$

This equation is well-known in fracture mechanic (Bui [42]; Guidera and Lardner [43]) and its solution is expressed as follows:

$$
\forall \underline{x} \in D \quad \omega_{3}(\underline{x})=\frac{2 d_{2}}{E(\lambda)} \underline{A} \cdot \underline{e}_{3} \sqrt{1-\underline{x} \cdot \boldsymbol{B} \cdot \underline{x}}
$$

where $\lambda=\sqrt{1-\left(\frac{d_{2}}{d_{1}}\right)^{2}}$ and $E(\lambda)$ is the complete elliptic integral of the second kind:

$$
E(\lambda)=\int_{\theta=0}^{\pi / 2} \sqrt{1-\lambda^{2} \sin ^{2} \theta} d \theta \quad(0 \leq \lambda \leq 1)
$$

The semi-analytical or iterative numerical expression of the complete elliptic integrals can be found in mathematical handbooks of Abramowitz and Stegun [44].

Considering the particular case of a circle of radius $d_{1}=d_{2}=R$, equation (24) reads:

$$
\forall \underline{x} \in D \quad \omega(\underline{x})=\frac{4}{\pi} \underline{A}_{\underline{e}} \underline{e}_{3} \sqrt{R^{2}-\underline{x} \cdot \underline{x}}
$$

By noting $\Psi$ the integral of discontinuity temperature function over the crack, we obtain:

$$
\Psi=\int_{D}[[T(\underline{x})]] \cdot \underline{n} d x=\frac{8}{3} R^{3}(\underline{n} \otimes \underline{n}) \cdot \underline{A}
$$

This closed-form solution is an important element to evaluate the effective thermal conductivity of cracked media by using the homogenization approaches. Three schemes, namely dilute, differential and self-consistent, will be considered in the section 4 .

\section{Thermal conductivity}

This section presents the homogenisation schemes to estimate the effective thermal conductivity of a medium embedding cracks that are non-conducting and acts as barriers to the heat flow. For this purpose, a domain $\Omega$, containing several cracks $\Gamma^{\mathrm{j}}$ prescribed on its boundary by a linear temperature $T=\underline{A} . \underline{x}$, i.e. a constant temperature gradient $\nabla T=\underline{A}$, is considered.

The average rate of heat flow and average temperature gradient over $\Omega$ are formulated as: 


$$
\underline{Q}=\frac{1}{\Omega} \int_{\Omega} \underline{q}(\underline{x}) d x ; \quad \underline{G}=\frac{1}{\Omega} \int_{\partial \Omega} T(\underline{x}) \cdot n(\underline{x}) d x=\underline{A}
$$

The effective thermal conductivity $\lambda^{\text {eff }}$ is given globally by Fourrier's law:

$$
\underline{Q}=-\lambda^{e f f} \cdot \underline{G}
$$

The divergence theorems for the temperature function in a domain containing discontinuities $\Gamma^{\mathrm{j}}$ reads:

$$
\int_{\Omega} \nabla T d x=\int_{\partial \Omega} T \cdot \underline{n} d x-\sum_{j} \int_{\Gamma^{j}}[[T]] \cdot \underline{n} d x
$$

Substituting equations (1) and (30) into the average rate of heat flow $Q$ yields

$$
\underline{Q}=-\lambda\left[\underline{A}-\frac{1}{\Omega} \sum_{j} \int_{\Gamma^{j}}[[T]] . \underline{n} d x\right]
$$

Equations (29) and (31) implies the reduction of thermal flow rate, by the temperature discontinuities across cracks $\frac{1}{\Omega} \sum_{j} \int_{\Gamma^{j}}[[T]] . \underline{n} d x$, is a linear function of average gradient of temperature $\underline{G}$, i.e. there exists a tensor $\Psi$ such as:

$$
\frac{1}{\Omega} \sum_{j} \int_{\Gamma^{j}}[[T]] . \underline{n} d x=\Psi \cdot \underline{G}
$$

Replacing $Q$ in equation (29) by its expression in equation (31) and (32) gives

$$
\lambda^{e f f}=\lambda(\boldsymbol{\delta}-\Psi)
$$

Therefore, the effective thermal conductivity can be determined when the temperature discontinuity on cracks are known. For the general case, solving equation (12) by numerical methods, such collocation method [20], Symmetry Garlerkin Boundary Element method [41], etc, allows obtaining the solution of temperature discontinuity field on cracks. Besides, the effective thermal conductivity $\lambda^{\text {eff }}$ can be estimated by using the theoretical up-scaling scheme based on the analytical solution (27). Three schemes are considered in the following and its results are then analyzed and compared with numerical solution in the literature.

\subsection{Dilute scheme}

Let us consider $m$ fracture families are embedded in a porous domain $\Omega$. Each family $\Gamma_{i}$ has a density $\rho_{\mathrm{i}}=N_{\mathrm{i}} / \Omega$ (i.e $N_{i}$ fractures within $\Omega$ ), orientation $\underline{n}_{\mathrm{i}}$ (unit normal vector) and 
radius $R_{\mathrm{i}}$ (Fig. 5). In the dilute framework, each crack is supposed to be subjected to the same far-field condition, i.e. undisturbed by the presence of other cracks. This approach presents a simple and accurate estimation of equivalent properties for material containing inhomogeneities when the crack density is small.

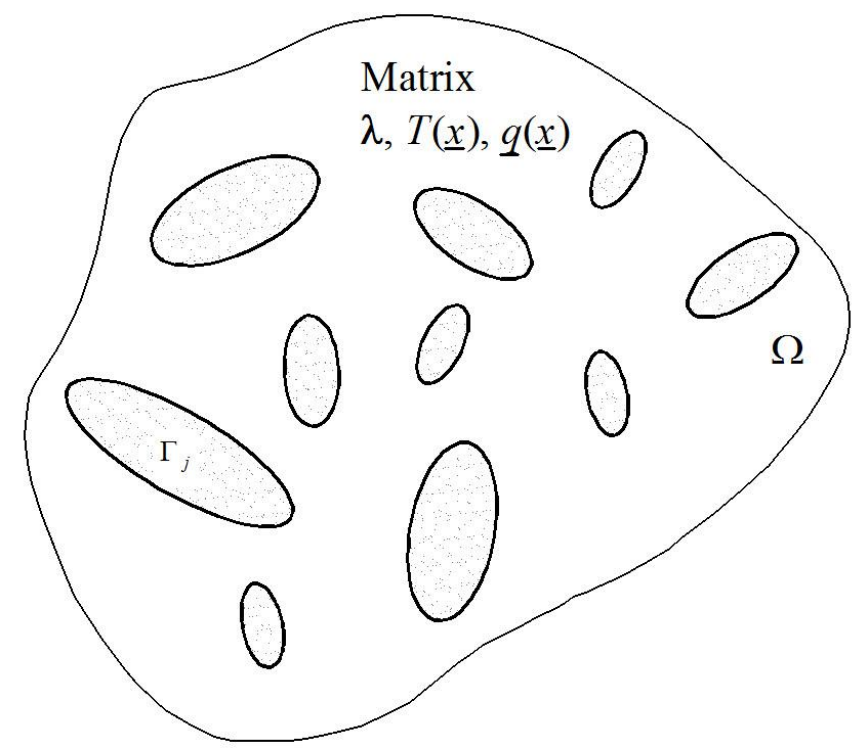

Fig. 5: Medium containing a weak crack density.

By neglecting fracture interaction, the term $\Psi$ in equation (32) is expressed such as:

$$
\Psi=\sum_{i}^{m} \frac{8}{3} \rho_{i} R_{i}^{3}\left(\underline{n}_{i} \otimes \underline{n}_{i}\right)
$$

The dimensionless crack density is introduced to cover other fracture shapes:

$$
v_{i}=\rho_{i} V_{0 i}
$$

In this relation, $V_{0 i}$ is a reference volume around a fracture of family $i$. Since the effective conductivity resulted in the current work will be compared to one obtained by Bogdanov et al. [14], we take thus $V_{0 i}=\pi^{2} R_{i}^{3}$ to unify the notation of dimensionless crack density between these two work. According to Adler and Thovert [27] and Charlaix et al. [28], for an isotropic distribution of fractures having the same circular shape of radius $R$, the volume $V_{e x}=\pi^{2} R^{3}$ is the excluded volume that is defined as one around an object of the system where other objects do not appear in order not to intersect it. As the exclusion volume characterizes all objects in the system as an ensemble, i.e. it is not given for each family independently, the volume $V_{0 i}$ does not have sense of excluded volume with the presence of several fracture families with different sizes and orientations. A general expression of $V_{\mathrm{ex}}$ for an anisotropic system could be referred in Mourzenko et al. $[29,30]$, where its calculation includes orientations, shapes and sizes of all fractions 
within the fracture network.

Substituting (35) into (34) yields:

$$
\Psi=\sum_{i}^{m} \frac{8}{3 \pi^{2}} v_{i}\left(\underline{n}_{i} \otimes \underline{n}_{i}\right)
$$

For the case of an isotropic distribution of fractures with density $v$ having identical

radius $R$, the average value $\langle\underline{n} \otimes \underline{n}\rangle=\frac{1}{3} \delta$, the effective conductivity tensor is isotropic $\lambda^{e f f}=\lambda^{e f f} \boldsymbol{\delta}$, and:

$$
\frac{\lambda^{\text {eff }}}{\lambda}=1-\frac{8}{9 \pi^{2}} v
$$

This formulation is identical to one given by Shafiro and Kachanov [47].

\subsection{Differential scheme}

It is worth noting that the crack density $\rho$ is a discrete function, however, the dimensionless crack density $v$ is a continuous function since the crack size, i.e $R$ varies from zero. Therefore, differential scheme can be applied to upscale the effective thermal conductivity of cracked materials. This method consists in estimate the thermal conductivity explicitly from an initial material through a series of incremental additions $[45,46]$. Supposing $d v$ the adding crack density, the effective thermal conductivity at a considered step is determined from one at the previous step by equation (37):

$$
\lambda_{i+1}=\lambda_{i}\left(1-\frac{8}{9 \pi^{2}} d v\right)
$$

Furthermore,

$$
\frac{\lambda_{i+1}-\lambda_{i}}{d v}=-\lambda_{i} \frac{8}{9 \pi^{2}}
$$

and then,

$$
\frac{d \lambda}{d v}=-\lambda \frac{8}{9 \pi^{2}}
$$

Integration the later gives:

$$
\lambda^{\text {eff }}=\lambda \exp \left(-\frac{8}{9 \pi^{2}} v\right)
$$

\subsection{Self-consistent scheme}


As seen in equation (31) the average rate of heat flow $Q$ is reduced by $Q^{*}=\lambda \Psi \underline{A}$ vis-a-vis one of un-cracked medium. Regardless the two previous approaches, the self-consistent scheme considers the cracks surrounding by matrix having the effective conductivity. In other words, $Q^{*}=\lambda^{e f f} \Psi \underline{A}$. Replacing $Q^{*}$ by this expression into (31) and then (29) yields:

$$
\frac{\lambda^{e f f}}{\lambda}=\frac{1}{1+\frac{8}{9 \pi^{2}} v}
$$

For the case $v \ll 1$, the first order expansion of equation (42) results the formulation (37). The results stemming from differential scheme (equation (41)) and self-consistent one (equation (41)) are compared with numerical result obtained by Bogdanov et al. [14]. This comparison allows choosing which theoretical approach is better in the case of media containing insulating crack.

Bogdanov et al. [14] investigated the effective property of finite porous media embedding an isotropic distribution of hexagonal fracture network based on a threedimensional finite volume method. Consider the insulating fracture have a conductivity $<1$ and a cross section resistance $>>1$. A least squares fit of all numerical results allows them to introduce an expression of effective property versus $v$ as follows:

$$
\frac{\lambda^{\text {eff }}}{\lambda}=1.0010-0.0604 v+0.0014 v^{2} \quad(v \leq 8)
$$

According to equations (37), (41), (42), (43), variations of effective thermal conductivities as function of crack density $v$ are presented in Fig. 6. Obviously, two approximated curves, namely differential model (41) and self-consistent one (42), are tangent to the curve of Shafiro and Kachanov [47] at $v=0$. It can be note that the selfconsistent model (42) is better than differential one (41) and diluted one (37) in comparison with numerical model of Bogdanov et al. [14]. 


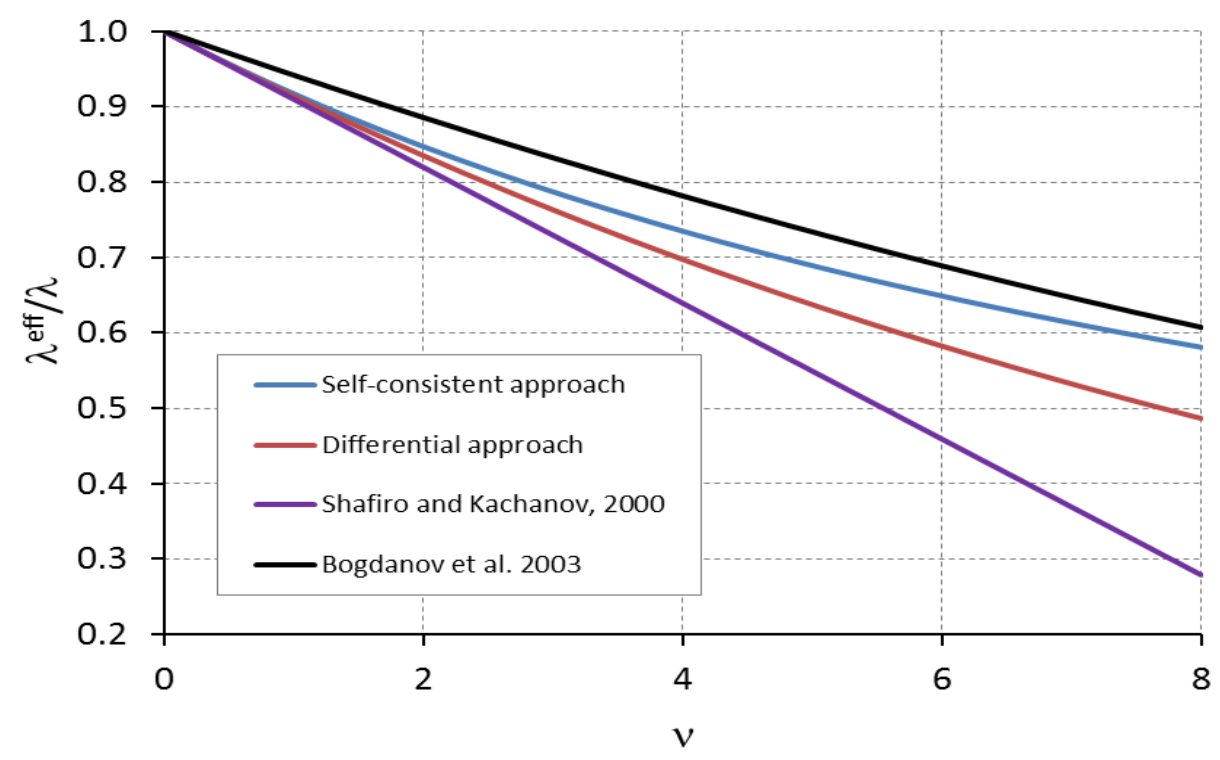

Fig. 6. Comparison homogenisation models of effective thermal conductivity of cracked medium (developed within this paper) with results obtained by using the finite volume approach proposed by Bogdanov et al. (2003).

In practical point of view, the characterization of crack system (size, distribution, orientation, connectivity) within a material is not an easy task, even impossible. Besides, the measured thermal conductivity on sample in laboratory and particularly in-situ is usually supposed to be isotropic, i.e. a randomly crack distribution. Therefore, the assumption of simple crack shape, as well as an isotropic cracks distribution is habitually made to simulate the experiment measurement and the real engineering processes. As a conclusion, although the current work considers a simple case of a system of equally sized disc shapes with isotropic distribution for cracks, but the selfconsistent model (42) with the validation against to numerical model [14] seems to be very useful in engineering fields. This model could be applied to estimate rapidly the thermal conductivity of cracked media or to determine the crack parameters from the measurement by inverse analysis.

\section{Conclusion}

Steady state heat transfer within media containing cracks, which act as barrier to heat flow, is investigated in this paper. The cracks are mathematically modelled by a two dimensional smooth surface of zero thickness. Multi-region boundary element method is used to obtain the potential solution that presents the temperature solution in the whole domain as function of thermal variables on the boundary, namely temperature and rate of heat flow, and temperature discontinuity on the cracks. This boundary integral equation 
allows reducing the dimension problem from 3D to 2D in numerical analysis. Fast multipoles method [48] dealing with this equation is on-going to model accurately the heat transfer within this material.

Applying the boundary integral equation to an infinite medium that contains a crack of elliptical shape, allows deriving the closed-form solution of temperature discontinuity across this crack surface. Based on this solution, three homogenization schemes (dilute, differential and self-consistent) are employed to estimate the effective thermal conductivity. The excluded volume is had recourse to extend the arbitrary form of crack. These three models are compared to numerical solution proposed in the literature to evaluating the validity of homogenization estimations. This comparison shows that the self-consistent model is the most appropriate to estimate the thermal conductivity of material containing insulating cracks.

\section{Acknowledgements}

The authors wish to thank the anonymous reviewers for their constructive comments on an earlier version of this article.

\section{References}

1. A. Redondo, J.G. Beery, Thermal conductivity of optical coatings, J. Appl. Phys. 60 (1986) 3882-3885.

2. S.E. Pestchanyi, I.S. Landman, Effective Thermal Conductivity of Graphite Materials with Cracks, Physica Scripta T111 (2004) 218-220.

3. R. Papitha, M Suresh, D Das, R Johnson, Effect of micro-cracking on the thermal conductivity and thermal expansion of tialite (A12TiO5) ceramics, Process. Appl. Ceram. 7 (2013) 143-146.

4. J.R. Nicholls, K.J. Lawson, A. Johnstone, D.S. Rickerby, Methods to reduce the thermal conductivity of EB-PVD TBCs, Surface and Coatings Technology 152 (2002) 383-391..

5. F. Cernuschi, S. Ahmaniemi, P. Vuoristo, T. Mäntylä, Modelling of thermal conductivity of porous materials: application to thick thermal barrier coatings, J. Eur. Ceram. Soc. 24 (2004) 2657-2667. doi:10.1016/j.jeurceramsoc.2003.09.012..

6. I. Sevostianov, M. Kachanov, J. Ruud, P. Lorraine, M. Dubois, Quantitative characterization of microstructures of plasma-sprayed coatings and their conductive and elastic properties, Mater. Sci. Eng. A. 386 (2004) 164-174. doi:10.1016/j.msea.2004.07.011..

7. T.J. Lu, J.W. Hutchinson, Thermal conductivity and expansion of cross-ply composites with matrix cracks, 43 (1995)..

8. L.-Q. Dao, P. Delage, A.-M. Tang, Y.-J. Cui, J.-M. Pereira, X.-L. Li, et al., Anisotropic thermal conductivity of natural Boom Clay, Appl. Clay Sci. 101 (2014) 282-287.

9. M. Bonnet, T. Burczyński, M. Nowakowski, Sensitivity analysis for shape perturbation of 
cavity or internal crack using BIE and adjoint variable approach. International Journal of Solids and Structures 39(9) (2002) 2365-2385.

10. M Bonnet, Higher-order topological sensitivity for 2-D potential problems. Application to fast identification of inclusions. International Journal of Solids and Structures 46(11-12) (2009) 2275-2292.

11. S.R. Stock, Recent advances in X-ray microtomography applied to materials, Int. Mater. Rev. 53 (2008) 129-181.

12. A. Pouya, M. Ghoreychi, Determination of rock mass strength properties by homogenisation, International Journal for Numerical and Analytical Methods in Geomechanics 25 (2001) 1285-1303.

13. P.N. Sævik, I. Berre, M. Jakobsen, M. Lien, A 3D Computational Study of Effective Medium Methods Applied to Fractured Media, Transport in Porous Media 100(1)(2013) 115-142.

14. I.I. Bogdanov, V.V. Mourzenko, J.F. Thovert, P.M. Adler, Effective permeability of fractured porous media in steady state flow, Water Resources Research 39 (2003) 1-16.

15. V.V. Mourzenko, I.I. Bogdanov, J.F. Thovert, P.M. Adler, Three-dimensional numerical simulation of single-phase transient compressible flows and well-tests in fractured formations, Mathematics and Computers in Simulation 81 (2010) 2270-2281.

16. I.I. Bogdanov, V.V. Mourzenko, J.F. Thovert, P.M. Adler, Effective permeability of fractured porous media with power-law distribution of fracture sizes, Phys. Rev. E 76 (2007) 036309.

17. V.V. Mourzenko, J.F. Thovert, P.M. Adler, Percolation and permeability of fracture networks in excavated damaged zones, Phys. Rev. E 86 (2012) 026312.

18. J.T. Katsikadelis: Boundary elements - Theory and Applications. Elsevier, 2002.

19. C.A. Brebbia, J. Dominguez, Boundary Elements: An Introduction Course. McGraw-Hill: New York, 1989.

20. M. Bonnet, Equations Intégrales et Éléments de Frontière. CNRS Editions/Eyrolles, Paris, 1995.

21. M.A. Atalay, E.D. Aydin, M. Aydin, Multi-region heat conduction problems by boundary element method, Int. J. Heat Mass Transf. 47 (2004) 1549-1553.

22. B. Shen, H.M. Kim, E.S. Park, T.K. Kim, M.W. Wuttke, M. Rinne, T. Backers, O. Stephansson, Multi-Region Boundary Element Analysis for Coupled Thermal-Fracturing Processes in Geomaterials, Rock Mechanics and Rock Engineering 46(1) (2013) 135-151.

23. A. Pouya, M.N. Vu, Numerical modelling of steady-state flow in 2D cracked anisotropic porous media by singular integral equations method, Transp. Porous Media 93(3) (2012) 475-493.

24. A. Pouya. Three-dimensional flow in fractured porous media: a potential solution based on singular integral equation, Adv. Water Resour. 35 (2012) 30-40.

25. A. Pouya, M.N Vu, Fluid flow and effective permeability of an infinite matrix containing disk-shaped cracks, Adv. Water Resour. 42 (2012) 37-46.

26. M.N. Vu, Modélisation des écoulements dans des milieux poreux fracturés par la méthode des équations intégrales singulières, PhD Thesis. Ecole des Ponts ParisTech 2012. 
27. P.M. Adler, J.F. Thovert, Fractures and Fracture Networks, Kluwer Acad.: Norwell, Mass.1999.

28. E. Charlaix, E. Guyon, N. Rivier, A criterion for percolation threshold in a random array of plates. Solid State Commun 50(11) (1984) 999-1002.

29. V.V. Mourzenko, J.F. Thovert, P.M. Adler, Trace analysis for fracture networks with anisotropic orientations and heterogeneous distributions, Phys. Rev. E 83 (2011) 031104.

30. V.V. Mourzenko, J.F. Thovert, P.M. Adler, Permeability of isotropic and anisotropic fracture networks, from the percolation threshold to very large densities, Phys. Rev. E 84 (2011) 036307.

31. I. Sevostianov, Thermal conductivity of a material containing cracks of arbitrary shape. International Journal of Engineering Science 44 (2006) 513-528.

32. A. Giraud, C. Gruescu, D.P. Do, F. Homand, D. Kondo, Effective thermal conductivity of transversely isotropic media with arbitrary oriented ellipsoïdal inhomogeneities, International Journal of Solids and Structures 44(9) (2007) 2627-2647.

33. C. Gruescu, A. Giraud, F. Homand, D. Kondo, D.P. Do, Effective thermal conductivity of partially saturated porous rocks, International Journal of Solids and Structures 44(3-4) (2007) 811-833.

34. D.P. Do, Application des approches d'homogénéisation à l'étude des propriétés thermohydro-mécaniques des roches. Application aux argilites, $\mathrm{PhD}$ thesis. Institut National Polytechnique de Lorraine (INPL), France 2008.

35. Q.D. To, G. Bonnet, A numerical-analytical coupling computational method for homogenization of effective thermal conductivity of periodic composites, Asia Pacific Journal on Computational Engineering 1(1) (2014) 1:5.

36. Q.D. To, G. Bonnet, V.T. To, Closed-form solutions for the effective conductivity of twophase periodic composites with spherical inclusions, Proceedings of the Royal Society A: Mathematical, Physical and Engineering Sciences, Royal Society 469(2151) (2013) 20120339.

37. S.T. Nguyen, Micromechanical approach for electrical resistivity and conductivity of sandstone, Journal of Applied Geophysics 111 (2014) 135-140.

38. Y. Chen, S. Zhou, R. Hu, C. Zhou, Estimating effective thermal conductivity of unsaturated bentonites with consideration of coupled thermo-hydro-mechanical effects, Int. J. Heat Mass Transf. 72 (2014) 656-667.

39. S.T. Nguyen, L. Dormieux, L.E. Yann, J. Sanahuja, A Burger model for the effective behavior of a microcracked viscoelastic solid, International Journal of Damage Mechanics 20(8) (2011) 1116-1129.

40. S.T. Nguyen, Generalized Kelvin model for micro-cracked viscoelastic materials. Engineering Fracture Mechanics 127 (2014) 226-234.

41. A Sutradhar, H.P. Glaucio, J.G. Leonard, Symmetric Galerkin Boundary Element Method. Springer, 2008.

42. H.D. Bui, Mécanique de la rupture fragile. Ed Masson, 1978.

43. J.T. Guidera, R.W. Lardner, Penny-shaped crack. Journal of Elasticity 5(1), (1975) 59-73.

44. M. Abramowitz, I.A. Stegun, (Eds.), The Process of the Arithmetic-Geometric Mean." $\S 17.6$ in Handbook of Mathematical Functions with Formulas, Graphs, and Mathematical 
Tables, 9th printing. New York: Dover, 598-599, 1972.

45. R. McLaughlin, A study of the differential scheme for composite materials, Int. J. Eng. Sci. 15(4) (1977) 237-244.

46. A.N. Norris, A differential scheme for the effective moduli of composites, Mech. Mater. 4 (1985) 1-16.

47. B. Shafiro, M. Kachanov, Anisotropic effective conductivity of material with nonrandomly oriented inclusions of divers ellipsoidal shapes, Journal of applied physics 87(12) (2000) 8561-8569.

48. Y. Liu, Fast Multipole Boundary Element Method: Theory and Applications in Engineering. Cambridge University Press, 2009. 